\title{
EUS-guided fiducial marker placement for radiotherapy in rectal cancer: feasibility of two placement strategies and four fiducial types
}

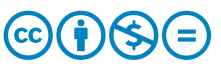

\author{
Authors \\ Monique E. van Leerdam ${ }^{1}$ \\ Institutions \\ 1 Department of Gastroenterology, the Netherlands \\ Cancer Institute, Amsterdam, The Netherlands \\ 2 Department of Radiotherapy, Leiden University Medical \\ Center, Leiden, The Netherlands \\ 3 Leiden Center for Interventional Endoscopy, \\ Department of Gastroenterology, Leiden University \\ Medical Center, Leiden, The Netherlands \\ 4 Department of Radiation Oncology, the Netherlands \\ Cancer Institute, Amsterdam, The Netherlands
}

Lisanne S. Rigter ${ }^{1}$, Eva C. Rijkmans², Akin Inderson ${ }^{3}$, Roy P.J. van den Ende², Ellen M. Kerkhof ${ }^{2}$, Martijn Ketelaars², Jolanda van Dieren ${ }^{1}$, Roeland A. Veenendaal ${ }^{3}$, Baukelien van Triest ${ }^{4}$, Corrie A.M. Marijnen ${ }^{2}$, Uulke A. van der Heide ${ }^{4}$,

submitted 3.10.2018

accepted after revision 3.12.2018

Bibliography

DOI https://doi.org/10.1055/a-0958-2148 |

Endoscopy International Open 2019; 07: E1357-E1364

(c) Georg Thieme Verlag KG Stuttgart · New York

elSSN 2196-9736

Corresponding author

Dr. M. E. van Leerdam, Department of Gastroenterology, Netherlands Cancer Institute, Plesmanlaan 121, 1066 CX Amsterdam

Fax: +31205122566

m.v.leerdam@nki.nl

\section{ABSTRACT}

Background and study aims To facilitate image guidance during radiotherapy of rectal cancer, we investigated the feasibility of fiducial marker placement. This study aimed to evaluate technical success rate and safety of two endoscopic ultrasound (EUS)-guided placement strategies and four fiducial types for rectal cancer patients.

Patients and methods This prospective multicenter study included 20 participants who were scheduled to undergo rectal cancer treatment with neoadjuvant short-course radiotherapy or chemoradiation. EUS-guided endoscopy was used for fiducial placement at the tumor site $(n=10)$ or in the mesorectal fat and in the tumor $(n=10)$. Four fiducial types were used (Visicoil $0.75 \mathrm{~mm}$, Visicoil $0.50 \mathrm{~mm}$, Cook, Gold Anchor). The endpoints were technical success rate and retention of fiducials, the latter of which was evaluated on cone-beam computed tomography scans during the first five radiotherapy fractions.

Results A total of 64 fiducials were placed in 20 patients. For each fiducial type, at least three fiducials were successfully placed in all patients. Technical failure consisted of fiducial blockage within the needle $(n=2)$ and ejection of two preloaded fiducials at once $(n=4)$. No serious adverse events were reported. In three patients, one of the fiducials was misplaced without clinical consequences; two in the prostate and one in the intraperitoneal cavity. After a median time of 17 days after placement (range 7-47 days), a total of $42 / 64$ (66\%) fiducials were still present (24/44 intratumoral vs. $18 / 20$ mesorectal fiducials, $P=0.009$ ).

Conclusions Placement of fiducials in rectal cancer patients is feasible, however, retention rates for intratumoral fiducials were lower $(55 \%)$ than for mesorectal fiducials (90\%).

\section{Introduction}

Neoadjuvant (chemo)radiotherapy, in addition to total mesorectal excision (TME), improves local control of rectal cancer [1-4]. However, patients experience long-term side effects after neoadjuvant (chemo)radiotherapy, including fecal incon- tinence and impaired sexual functioning [5-8]. A reduction in target volume may reduce these side effects. However, precise irradiation of the target remains difficult for rectal cancer due to tumor motion and poor visibility of the tumor area on conebeam CT (CBCT). Fiducial markers may improve radiotherapy 
position verification, not only for external beam radiotherapy, but more importantly for brachytherapy.

Prior studies evaluated endoluminal clips for this purpose, demonstrating limited usefulness due to poor long-term retention rates ranging from $50 \%$ to $75 \% 1$ week after placement [ 9 , 10]. Preferably at least two clips should remain present in a patient during the full course of radiotherapy. In addition, these endoluminal clips create large artefacts on magnetic resonance imaging (MRI).

As an alternative, MRI-compatible fiducials may be used, since they have adequate retention rates after implantation in many solid cancers, such as prostate, esophageal, and pancreatic cancer [11-13]. Three previous studies described successful placement of fiducials in rectal cancers, in 54, 11, and 9 patients, respectively [13-15]. In these studies, different placement techniques and different fiducial types were used. One of these studies evaluated postprocedural loss of intratumoral fiducials, resulting in loss of 10 of 39 fiducials during radiotherapy [14]. The optimal placement technique and fiducial type have thus not been identified.

Usefulness of rectal cancer fiducials is strongly dependent on the rate of retention of the fiducials, on visibility on images used for target delineation and treatment planning, and on visibility on CBCT scans.

This pilot study aimed to evaluate technical feasibility and safety of EUS-guided fiducial placement at the tumor site in patients with rectal cancer, and fiducial loss after placement, comparing two fiducial placement strategies and four different fiducial types.

\section{Patients and methods \\ Study design and population}

In this prospective interventional pilot study we included 20 rectal cancer patients in the Netherlands Cancer Institute (NKI) and Leiden University Medical Center (LUMC). Participants were to be treated for rectal cancer with short-course radiotherapy (5 $\times 5$ Gy) or chemoradiation consisting of $25 \times 2$ Gy combined with capecitabine $825 \mathrm{mg} / \mathrm{m}^{2}$ twice daily, followed by total mesorectal excision. Exclusion criteria were coagulopathy, use of anticoagulants (vitamin K antagonists, direct oral anticoagulants), prior pelvic irradiation or surgery, World Health Organization performance status 3-4, pregnancy, prior hip replacement, or a contraindication for MRI.

The study procedure included an endoscopic ultrasound (EUS)-guided endoscopy with placement of fiducials. If no clear EUS view could be obtained, a forward-looking endoscope was used and fiducials were placed under direct view into the tumor.

The study protocol was approved by the medical ethics committee of the Netherlands Cancer Institute and the study was registered at the Dutch Trial Registry (trial ID NTR4606). All participating patients provided written informed consent.

\section{Fiducial marker placement}

At least 1 day before the first fraction of radiotherapy, all patients received a phosphate enema followed by EUS-guided endoscopy of the rectum with placement of three fiducials. Fiducial placement was performed by four experienced gastroenterologists, two in each study center. Four types of fiducials were used in this study (Visicoil $0.75 \mathrm{~mm} \times 5 \mathrm{~mm}$ and Visicoil $0.50 \mathrm{~mm} \times 5 \mathrm{~mm}$ [IBA Dosimetry GmbH, Germany], Cook $0.64 \mathrm{~mm} \times 3.4 \mathrm{~mm}$ [Cook Medical, Limerick, Ireland] and Gold Anchor $0.28 \mathrm{~mm} \times 20 \mathrm{~mm}$ [unfolded length, Naslund Medical $A B$, Sweden]). All fiducials were certified by the European Conformity (CE). Attribution of a fiducial type to a participant in each study center was performed randomly.

EUS was performed using a linear-array endoechoscope (Pentax, EG-3270UK, Pentax, EG-3870UTK, Olympus GIF-Q180, Olympus GIF-H180, or Fujinon, EG-580UT). The target lesion was visualized and absence of intervening vascular structures was ascertained. A fine-needle aspiration EUS needle (19 gauge or 22 gauge, Cobra Medical or Cook EchoTip Ultra) was inserted into the target area under EUS guidance or direct endoscopic view. The EUS needle was loaded with one of the fiducials and the tip was sealed with sterile bone wax. The Cook EchoTip Ultra Fiducial Needle was pre-loaded with four fiducials.

Two strategies for fiducial placement were evaluated. In the first 10 patients, defined as group 1 , three fiducials were placed into the tumor (one proximal, one central and one distal). In the second 10 patients, defined as group 2, we aimed to place at least two fiducials in the mesorectal fat (one proximal and one distal from the tumor) and one fiducial in the center of the tumor.

\section{Periprocedural care}

Periprocedural medication was not administered in participating patients (no sedatives, analgesia or prophylactic antibiotics were given). Patients were instructed to contact the radiation oncologist at any sign of fever, a change in pain or other unexpected adverse reactions. Patients were monitored by the radiation oncologist during regular outpatient clinic appointments during and after (chemo)radiotherapy.

\section{Outcome measures}

Technical success was defined as placement of three fiducials at the desired location in the rectum. Technical feasibility also included technical failure and technical difficulty of the EUS procedure, and second fiducial loss during radiotherapy. "Technical failure" comprised fiducial loading or unloading problems, whereas "technical difficulty" included problems with identifying tumor and surrounding tissue, which limits obtainment of a safe window for fiducial placement at the desired location, or inability to visualize the fiducials after insertion by EUS. Fiducial loss was evaluated by planning CT scans (when available) and over the course of radiotherapy by assessing the fiducials on CBCTs.

Adverse events (AEs) included any undesirable experience that occurred to a patient during the study, defined as the period between placement of the fiducials andI TME or a maximum 
of 30 days follow-up, whether or not considered related to the experimental intervention.

\section{Statistical analyses}

Data were analyzed in IBM SPSS Statistics 22. Patient and tumor characteristics and differences in fiducial retention were compared between groups using Chi Square or Fishers Exact tests.

\section{Results}

\section{Patient and tumor characteristics}

Participants were included between June 2015 and September 2016. Rectal cancer treatment consisted of neoadjuvant shortcourse radiotherapy in 11 patients and chemoradiation in nine patients. In one patient, a complete response was seen after chemoradiation and a wait and see policy was adopted. Median age at diagnosis was 62 years (range $51-82$ years). Two of 20 patients used a platelet aggregation inhibitor, which was continued during fiducial placement.

In the first 10 patients (group 1), fiducials were only placed at the tumor site. In the second 10 patients (group 2), fiducials were aimed to be placed in both the mesorectal fat and the tumor. No clear differences were found in baseline characteristics of these two patient groups, including age, gender, or TNM stage ( $\triangleright$ Table 1 ). Patients in group 2 , with fiducials aimed for the mesorectum (and tumor), appeared to receive more frequent treatment with chemoradiation.

\section{Feasibility of EUS-guided fiducial placement}

\section{Technical success}

A total of 64 fiducials were placed in 20 patients ( $>$ Table 2 ). In group 1 , at least three fiducials were successfully placed in the tumor of each patient.

In nine of 10 patients in group 2 , fiducials were placed in the mesorectal fat, including eight patients with at least two fiducials in mesorectal fat ( $\triangleright$ Table $\mathbf{1}$ ). In one tumor, only one fiducial could be placed in the mesorectal fat, because surrounding tissues limited the options for a safe window of placement of a second fiducial in the mesorectal fat. Placement of a fiducial in the mesorectal fat both proximal and distal from the tumor was feasible in only three of 10 patients. Placement of fiducials in the mesorectal fat was limited to proximal from the tumor in another three of 10 patients, because the tumor was close to the anal verge. In the final three of 10 patients, the tumor could not be passed by the endoscope and the fiducials in the mesorectal fat were placed only distal from the tumor.

\section{Technical failure}

Unloading problems occurred during placement of six fiducials. During placement of Cook fiducials, two fiducials were ejected at once in four patients. In one other patient, two of three Gold Anchors inserted in $19 \mathrm{G}$ needles consecutively blocked within the sheet of the needle and could not be removed. All other Gold Anchor fiducials were placed with a $22 \mathrm{G}$ needle without any problems.

\section{Technical difficulty}

The overview obtained by EUS was limited in seven patients. In five of them with intratumoral fiducials, it was not feasible to obtain clear delineation of the small tumor by EUS for all fiducials and at least one fiducial in these five patients was placed under direct vision with forward-looking endoscopy into the tumor. In another patient with the aim of placing fiducials in the mesorectal fat, it was not feasible to create a safe window for fiducial placement into this area, resulting in placement of three fiducials at the tumor site. In the third patient with a limited overview by EUS, the endoscopist noted that identification of the prostate and surrounding tissues was unclear. Indeed, one of the fiducials was placed in the prostate in this patient. In two additional patients, CBCT displayed a location of one of the fiducials outside the mesorectum. This included one patient with a fiducial in the prostate. The other patient had a proximal rectal cancer, and a fiducial was present in the peritoneal cavity which was not observed during EUS. None of these patients showed any signs or symptoms that had a probable relation to the procedure, and treatment was completed as planned.

EUS visualization of the placed fiducial was evaluated in 10 of 20 patients ( $\mathbf{F i g .} \mathbf{1}$ ). In five of 10 evaluated patients, not all three fiducials were visible by EUS.

\section{Fiducial loss evaluated on CBCTs}

CBCT scans for radiotherapy treatment planning and positioning were used for evaluation of fiducial loss.

Median time between fiducial placement and the first fraction of radiotherapy was 6 days (range $1-18$ days). On the first CBCT, 43 of 64 (67\%) of the presumably successfully placed fiducials were visible ( $\triangleright$ Fig.2). Only one additional fiducial was lost during radiotherapy. Median time between fiducial placement and the last CBCT was 17 days (range 7-47 days), after which 42 of 64 (66\%) fiducials were detected. In all patients, at least one fiducial was present at the end of follow-up.

When comparing fiducial retention rates based on placement location per fiducial, $55 \%$ of intratumoral fiducials were still detected on the final CBCT (detected in group 1 and group 2 , retention rates ranged from $46 \%$ to $67 \%$ between fiducial types) compared with $90 \%$ in mesorectal fiducials (group 2 only, Fishers' exact $P=0.009$, which ranged from $50 \%$ to $100 \%$ between fiducial types) ( $\triangleright$ Table 3 ).

Additional comparison of placement strategies between groups demonstrated a retention rate of 15 of 32 (47\%) fiducials in group 1 (intratumoral fiducials only) and 27 of 32 (84\%) fiducials in group 2 (both in the mesorectal fat and intratumoral, $P=0.002)$.

\section{Patient safety}

No serious AEs were reported. During approximately 1 week post-fiducial placement, symptoms consisted of an increase in blood loss in stool $(n=3)$ and in flatulence $(n=5)$. 
- Table 1 Characteristics of two patient groups with different fiducial placement strategies.

\begin{tabular}{|c|c|c|}
\hline Baseline characteristics & $\begin{array}{l}\text { Group 1: Patients with fiducials aimed } \\
\text { for the tumor }(\mathrm{N})\end{array}$ & $\begin{array}{l}\text { Group 2: Patients with fiducials aimed } \\
\text { for mesorectum (and tumor) (N) }\end{array}$ \\
\hline $\begin{array}{l}\text { Age } \\
\text { (median, range in years) }\end{array}$ & $65(57-82)$ & $60(51-65)$ \\
\hline \multicolumn{3}{|l|}{ Gender } \\
\hline - Male & 8 & 7 \\
\hline - Female & 2 & 3 \\
\hline \multicolumn{3}{|l|}{ Tstage } \\
\hline . $\mathrm{T} 2$ & 2 & 2 \\
\hline - $\mathrm{T3}$ & 8 & 8 \\
\hline \multicolumn{3}{|l|}{ N stage } \\
\hline . N0 & 4 & 3 \\
\hline - $\mathrm{N}+$ & 6 & 7 \\
\hline $\begin{array}{l}\text { Endoscopic distance from anal verge } \\
\text { (median, range in } \mathrm{cm} \text { ) }\end{array}$ & $8(0-15)$ & $6(1-16)$ \\
\hline \multicolumn{3}{|l|}{ Treatment } \\
\hline $.5 \times 5$ & 7 & 4 \\
\hline . CRT & 3 & 6 \\
\hline \multicolumn{3}{|l|}{ Fiducial placement characteristics } \\
\hline \multicolumn{3}{|l|}{ Fiducial type } \\
\hline - Visicoil 0.50 & 3 & 2 \\
\hline - Visicoil 0.75 & 4 & 1 \\
\hline - Cook & 3 & 2 \\
\hline - Gold Anchor & 0 & 5 \\
\hline \multicolumn{3}{|l|}{ Fiducial location } \\
\hline - Intratumoral only & 10 & 1 \\
\hline \multicolumn{3}{|l|}{ Mesorectal fiducials: number } \\
\hline .1 & & 1 \\
\hline . $\geq 2$ & & 8 \\
\hline \multicolumn{3}{|c|}{ Mesorectal fiducials: location in relation to tumor } \\
\hline - Proximal $(\geq 1) \&$ distal $(\geq 1)$ & & 3 \\
\hline - Proximal ( $\geq 1$, not distal) & & 3 \\
\hline - Distal ( $\geq 1$, not proximal) & & 3 \\
\hline
\end{tabular}

\section{Discussion}

This prospective multicenter study was the first to compare two fiducial placement strategies for rectal cancer to evaluate technical feasibility and fiducial retention rates. We demonstrated that fiducial retention rates are higher when fiducials are placed in the mesorectal fat instead of in the tumor. Because of the higher retention rate of mesorectal fiducials, this strate- gy appears more useful for position verification in image-guided radiotherapy or brachytherapy. Intratumoral fiducial placement was considered especially difficult in smaller tumors with limited volume for fiducial placement. Placement of all four investigated fiducial types was feasible.

Prior studies on endoluminal clips in rectal cancer were disappointing, due to intraluminal movement of the clips, poor long-term retention rates (ranging from 50\% to $75 \% 1$ week 


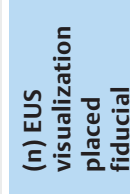

mil

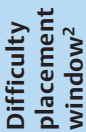

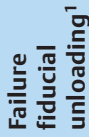

岀

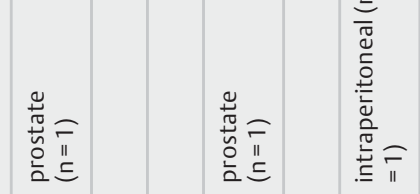

要

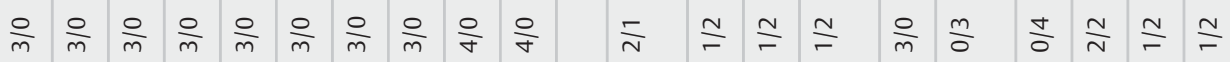

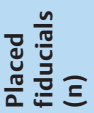

을

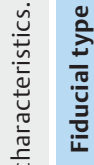

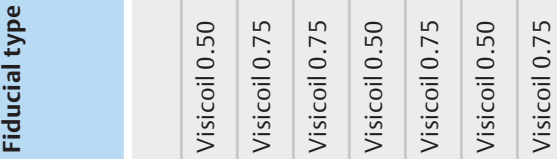

$g, g a g z a z a$

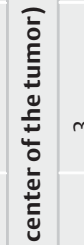

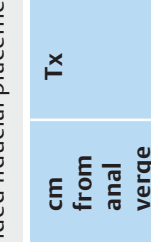

崖

跣

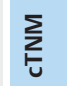

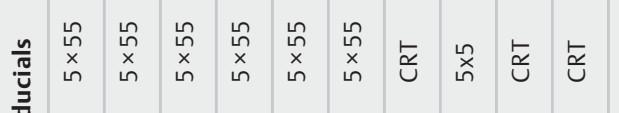

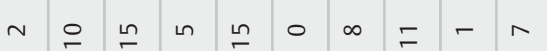

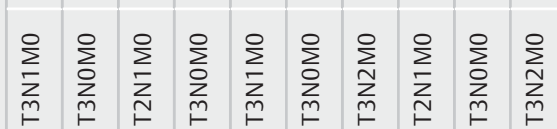

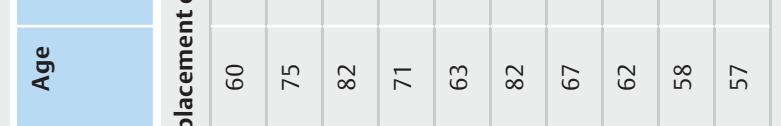

跣

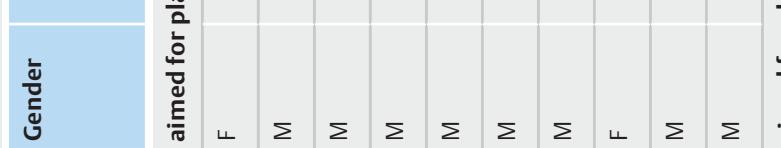

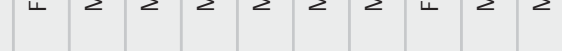
- amanon a o 0 


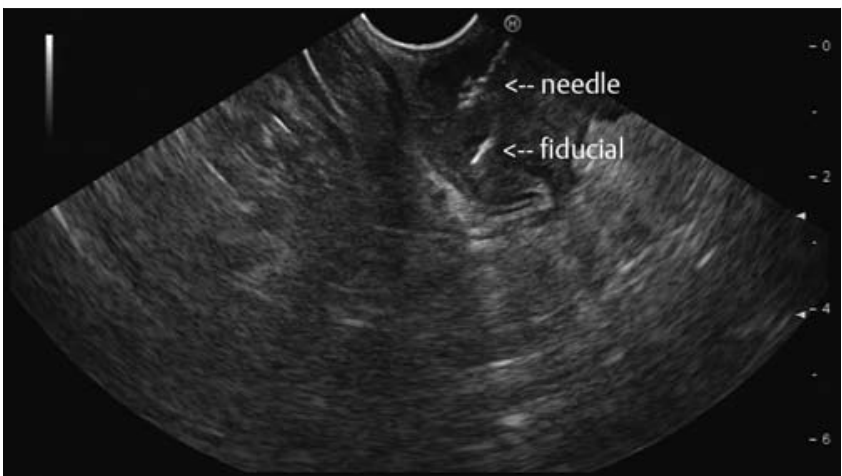

Fig. 1 Fiducial placed under EUS-guidance.

after placement) and MRI artifacts caused by the clips $[9,10]$. This led to exploration of the feasibility of fiducials, as they are more frequently compatible with MRI and appear to stay in place in other organs. A first report by Vorwerk et al. on rigid rectoscopy for placement of fiducials in the mesorectal tissue of nine patients with rectal cancer demonstrated $100 \%$ retention rates in the first 5 weeks after placement [15]. A consecutive study of EUS-guided endoscopic placement of intratumoral fiducials in 11 patients resulted in a fiducial retention rate of $74 \%$ at the time of surgery [14].

In our study, only $55 \%$ of intratumoral fiducials were present on CBCT after a median follow-up of 17 days, in comparison with $90 \%$ of fiducials placed in the mesorectal fat. In seven patients with intratumoral fiducials, only one fiducial was present at the end of the first week of radiotherapy. This limits the usefulness of the fiducials, as the presence of at least two fiducials is necessary for interpretation of the location of fiducials in re- lation to the tumor, especially when taking rectal motion into account.

Intratumoral placement of fiducials was challenged by the small volume and the soft consistency of the tumor. In addition, placement of fiducials in the mesorectal fat was associated with some technical challenges. It was considered difficult to obtain a safe window for mesorectal fiducial placement, due to surrounding tissues such as the prostate, seminal vesicles, bladder, vessels, and lymph nodes. This limited window may have led placement of three fiducials outside the mesorectal fat. Unfortunately, not all fiducials were visible by EUS after insertion, which limited confirmation of placement locations. No other AEs were described.

In the study by Vorwerk et al., who described fiducial placement in the mesorectum in nine patients, a fiducial located in the peritoneum was detected in one of nine patients [15]. In another study using EUS-guided endoscopy for intratumoral fiducial placement, one minor bleed and one undefined technical difficulty were described in a total of 54 patients [13]. The oncologic and non-oncologic health risks of fiducial placement in (or migration to) other tissues than the (meso)rectum appear low, as no symptoms were reported and treatment was finalized as planned. No evidence exists for routine administration of prophylactic antibiotics, as were given in the study by Moningi et al [14].

We evaluated four different types of fiducials, which were all successfully inserted at the desired location. There was no clear difference between the feasibility of the four types, however, use of Cook fiducials more frequently led to simultaneous insertion of two fiducials at once, and Gold Anchor fiducials blocked twice within the $19 \mathrm{G}$ needle. EUS visibility of fiducials after placement appeared more difficult when using smaller fiducials. We did not find a clear difference in retention rates be-

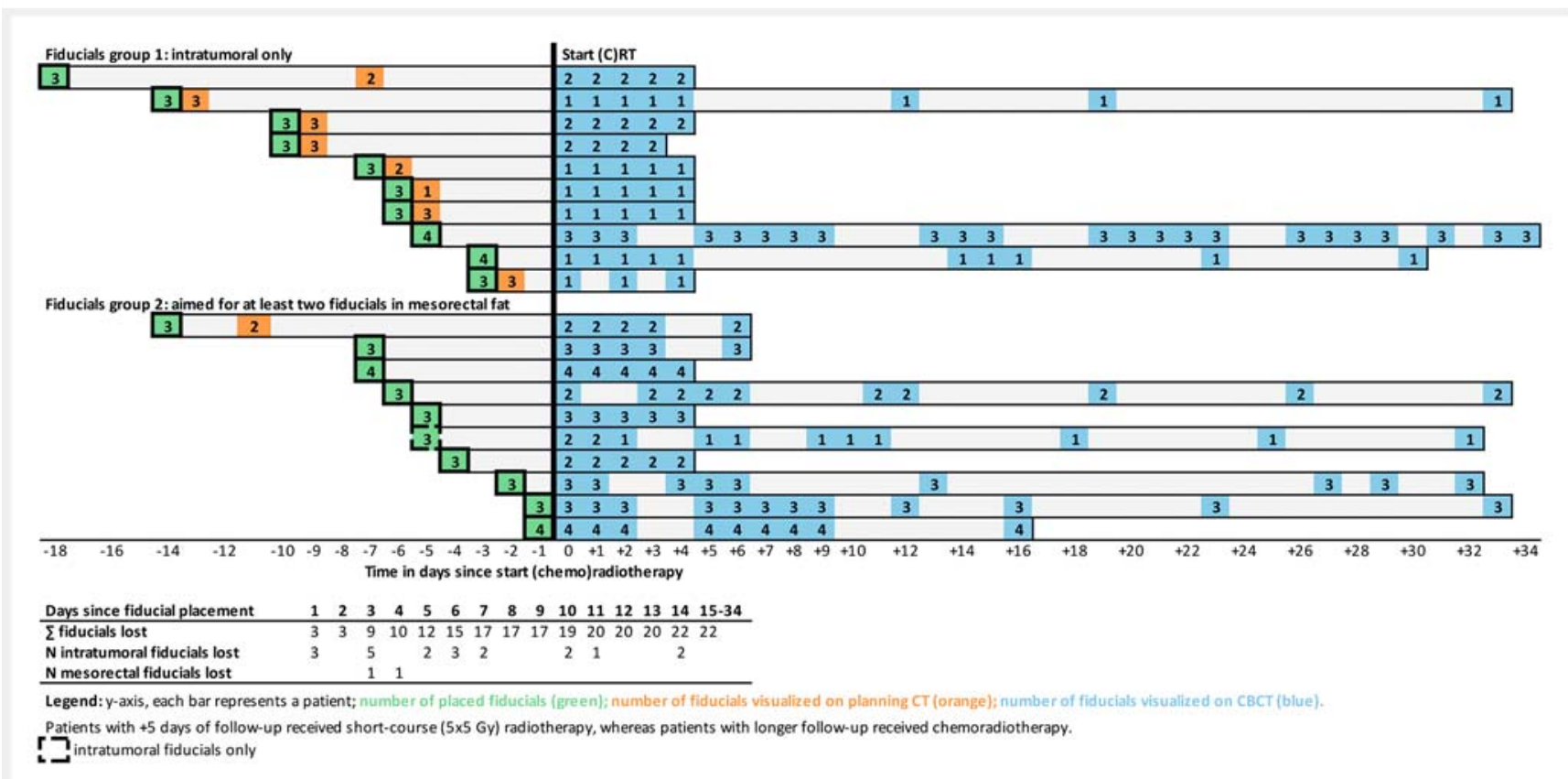

Fig. 2 Fiducial detection by CBCTs after placement. 
- Table 3 Fiducial characteristics: description of four fiducial types.

\begin{tabular}{|l|l|l|l|l|l|}
\hline Characteristic & Total & Visicoil $\mathbf{0 . 5 0}$ & Visicoil $\mathbf{0 . 7 5}$ & $\begin{array}{l}\text { Cook } \\
\text { N }\end{array}$ & $\begin{array}{l}\text { Gold Anchor } \\
\text { N (\%) }\end{array}$ \\
\hline Placed fiducials & N (\%) & 15 & 19 & 15 \\
\hline - Retained at end of follow-up & $42(66)$ & $9(60)$ & $7(47)$ & $14(74)$ & $12(80)$ \\
\hline Intratumoral fiducials & $44(69)$ & $12(80)$ & $13(87)$ & $13(68)$ & $6(40)$ \\
\hline - Retained at end of follow-up & $24(55)$ & $6(50)$ & $6(46)$ & $8(62)$ & $4(67)$ \\
\hline Mesorectal fiducials & $20(31)$ & $3(10)$ & $2(13)$ & $6(32)$ & $9(60)$ \\
\hline - Retained at end of follow-up & $18(90)$ & $3(100)$ & $1(50)$ & $6(100)$ & $8(89)$ \\
\hline
\end{tabular}

tween fiducial types, as this appeared more likely related to the location of fiducial placement. Future studies may explore the option of MRI-guided brachytherapy, which may lead to a preference of a fiducial depending on MRI visibility and migration properties.

In other gastrointestinal tumor locations, such as the esophagus and the pancreas, fiducials are more widely investigated and used $[13,16-19]$. Retention rates for fiducials placed in the tumor or surrounding tissue in esophageal and pancreatic cancer are $66 \%$ to $94 \%$ and $93 \%$ to $100 \%$, respectively [17, 20 -24]. The relatively high rate of intratumoral fiducial loss in rectal cancer may be due to a small tumor volume, rectal motion or the passing of stool [25].

\section{Conclusion}

In conclusion, EUS-guided placement of fiducials for rectal cancer is feasible and safe, but adequate positioning remains a challenge. Placement of fiducials in the mesorectal fat leads to a higher rate of retention of fiducials, however, these results could be influenced by other factors (e.g. fiducial type) and should be confirmed in a larger study.

\section{Acknowledgements}

The authors thank all patients and their families participating. In addition, they would like to thank Corine de Jong, Anja Betgen, Tineke Vijlbrief and Vera Patan for their logistic assistance.

\section{Competing interests}

Cook Medical fiducials were provided without cost. Visicoil and Gold Anchor fiducials were funded by Hypels van der Zee Fons (grant 3217/28-3-13/NZ). The producers of the fiducials had no influence on the design or execution of the trial. This study was partly funded by the Dutch Cancer Society, grant UL2013-6311 and Leiden University Fund (LUF)/Nypels van der Zee Fonds (grant 3217/28-3-13/NZ).

\section{References}

[1] van Gijn W, Marijnen CA, Nagtegaal ID et al. Preoperative radiotherapy combined with total mesorectal excision for resectable rectal cancer: 12-year follow-up of the multicentre, randomised controlled TME trial. Lancet Oncol 2011; 12: 575-582

[2] Sebag-Montefiore D, Stephens RJ, Steele R et al. Preoperative radiotherapy versus selective postoperative chemoradiotherapy in patients with rectal cancer (MRC CR07 and NCIC-CTG C016): a multicentre, randomised trial. Lancet 2009; 373: 811-820

[3] Sauer R, Becker H, Hohenberger W et al. Preoperative versus postoperative chemoradiotherapy for rectal cancer. N Engl J Med 2004; 351: $1731-1740$

[4] Peeters KC, Marijnen CA, Nagtegaal ID et al. The TME trial after a median follow-up of 6 years: increased local control but no survival benefit in irradiated patients with resectable rectal carcinoma. Ann Surg 2007; 246: $693-701$

[5] Dahlberg M, Glimelius B, Graf W et al. Preoperative irradiation affects functional results after surgery for rectal cancer: results from a randomized study. Dis Colon Rectum 1998; 41: 543 - 549; discussion 549-551

[6] Marijnen CA, van de Velde C], Putter $\mathrm{H}$ et al. Impact of short-term preoperative radiotherapy on health-related quality of life and sexual functioning in primary rectal cancer: report of a multicenter randomized trial. J Clin Oncol 2005; 23: 1847-1858

[7] Birgisson H, Pahlman L, Gunnarsson U et al. Occurrence of second cancers in patients treated with radiotherapy for rectal cancer. J Clin Oncol 2005; 23: 6126-6131

[8] Peeters KC, van de Velde C], Leer JW et al. Late side effects of shortcourse preoperative radiotherapy combined with total mesorectal excision for rectal cancer: increased bowel dysfunction in irradiated patients-a Dutch colorectal cancer group study. J Clin Oncol 2005; 23: 6199-6206

[9] Swellengrebel HA, Marijnen CA, Vincent A et al. Evaluating long-term attachment of two different endoclips in the human gastrointestinal tract. World J Gastrointest Endosc 2010; 2: 344-348

[10] Vuong T, Devic S, Moftah B et al. High-dose-rate endorectal brachytherapy in the treatment of locally advanced rectal carcinoma: technical aspects. Brachytherapy 2005; 4: 230-235

[11] Davila Fajardo R, Lekkerkerker SJ, van der Horst A et al. EUS-guided fiducial markers placement with a 22-gauge needle for image-guided radiation therapy in pancreatic cancer. Gastrointest Endosc 2014; 79 : $851-855$

[12] Moman MR, van der Heide UA, Kotte AN et al. Long-term experience with transrectal and transperineal implantations of fiducial gold markers in the prostate for position verification in external beam radio- 
therapy; feasibility, toxicity and quality of life. Radiother Oncol 2010; 96: $38-42$

[13] Dhadham GC, Hoffe S, Harris CL et al. Endoscopic ultrasound-guided fiducial marker placement for image-guided radiation therapy without fluoroscopy: safety and technical feasibility. Endosc Int Open 2016; 4: E378-E382

[14] Moningi S, Walker AJ, Malayeri AA et al. Analysis of fiducials implanted during EUS for patients with localized rectal cancer receiving highdose rate endorectal brachytherapy. Gastrointest Endosc 2015; 81: $765-769$ e761

[15] Vorwerk H, Liersch T, Rothe $\mathrm{H}$ et al. Gold markers for tumor localization and target volume delineation in radiotherapy for rectal cancer. Strahlenther Onkol 2009; 185: 127-133

[16] Pishvaian AC, Collins B, Gagnon G et al. EUS-guided fiducial placement for CyberKnife radiotherapy of mediastinal and abdominal malignancies. Gastrointest Endosc 2006; 64: 412-417

[17] Fernandez DC, Hoffe SE, Barthel JS et al. Stability of endoscopic ultrasound-guided fiducial marker placement for esophageal cancer target delineation and image-guided radiation therapy. Pract Rad Oncology 2013; 3: $32-39$

[18] de Souza Lawrence L, Ford E, Gilbert C et al. Novel applications of an injectable radiopaque hydrogel tissue marker for management of thoracic malignancies. Chest 2013; 143: 1635-1641

[19] Park WG, Yan BM, Schellenberg D et al. EUS-guided gold fiducial insertion for image-guided radiation therapy of pancreatic cancer: 50 successful cases without fluoroscopy. Gastrointest Endosc 2010; 71: $513-518$

[20] Machiels M, van Hooft J, jin P et al. Endoscopy/EUS-guided fiducial marker placement in patients with esophageal cancer: a comparative analysis of 3 types of markers. Gastrointest Endosc 2015; 82: 641 649

[21] Choi JH, Seo DW, Park DH et al. Fiducial placement for stereotactic body radiation therapy under only endoscopic ultrasonography guidance in pancreatic and hepatic malignancy: practical feasibility and safety. Gut Liver 2014; 8: 88-93

[22] Varadarajulu S, Trevino JM, Shen $\mathrm{S}$ et al. The use of endoscopic ultrasound-guided gold markers in image-guided radiation therapy of pancreatic cancers: a case series. Endoscopy 2010; 42: 423-425

[23] Sanders MK, Moser A], Khalid A et al. EUS-guided fiducial placement for stereotactic body radiotherapy in locally advanced and recurrent pancreatic cancer. Gastrointest Endosc 2010; 71: 1178-1184

[24] Khashab MA, Kim KJ, Tryggestad E] et al. Comparative analysis of traditional and coiled fiducials implanted during EUS for pancreatic cancer patients receiving stereotactic body radiation therapy. Gastrointest Endosc 2012; 76: 962 - 971

[25] Brierley JD, Dawson LA, Sampson E et al. Rectal motion in patients receiving preoperative radiotherapy for carcinoma of the rectum. Int J Radiat Oncol Biol Phys 2011; 80: 97-102 